\title{
Article
}

\section{Dynamic contrast-enhanced computed tomography for the diagnosis of solitary pulmonary nodules: a systematic review and meta-analysis}

Weir-McCall, Jonathan R., Joyce, Stella, Clegg, Andrew, MacKay, James W., Baxter, Gabrielle, Dendl, Lena-Marie, Rintoul, Robert C., Qureshi, Nagmi R., Miles, Ken and Gilbert, Fiona J.

Available at http://clok.uclan.ac.uk/31894/

Weir-McCall, Jonathan R., Joyce, Stella, Clegg, Andrew ORCID: 0000-00018938-7819, MacKay, James W., Baxter, Gabrielle, Dendl, Lena-Marie, Rintoul, Robert C., Qureshi, Nagmi R., Miles, Ken et al (2020) Dynamic contrastenhanced computed tomography for the diagnosis of solitary pulmonary nodules: a systematic review and meta-analysis. European Radiology . ISSN 0938-7994

It is advisable to refer to the publisher's version if you intend to cite from the work. http://dx.doi.org/10.1007/s00330-020-06661-8

For more information about UCLan's research in this area go to http://www.uclan.ac.uk/researchgroups/ and search for <name of research Group>.

For information about Research generally at UCLan please go to http://www.uclan.ac.uk/research/

All outputs in CLoK are protected by Intellectual Property Rights law, including Copyright law. Copyright, IPR and Moral Rights for the works on this site are retained by the individual authors and/or other copyright owners. Terms and conditions for use of this material are defined in the policies page. 
Dynamic contrast enhanced computed tomography for the diagnosis of solitary pulmonary nodules: A systematic review and meta-analysis. 


\begin{abstract}
Introduction: A systematic review and meta-analysis were performed to determine the diagnostic performance of dynamic contrast enhanced computed tomography (DCE-CT) for the differentiation between malignant and benign pulmonary nodules.
\end{abstract}

Methods: Ovid MEDLINE and EMBASE were searched for studies published up to October 2018 on the diagnostic accuracy of DCE-CT for the characterization of pulmonary nodules. For the index test, studies with a minimum of a pre- and post-contrast computed tomography scan were evaluated. Studies with a reference standard of biopsy for malignancy, and biopsy or 2-year follow-up for benign disease were included. Study bias was assessed using QUADAS-2 (Quality Assessment of Diagnostic Accuracy Studies). The sensitivities, specificities, and diagnostic odds ratios were determined along with $95 \%$ confidence intervals (Cls) using a bivariate random effects model.

Results: Twenty-three studies were included, including 2397 study participants with 2514 nodules with $55.3 \%$ malignancy $(1389 / 2514)$. The pooled accuracy results were: sensitivity $94.8 \%(95 \% \mathrm{Cl}$ 91.5;96.9), specificity 75.5\% (69.4;80.6), diagnostic odds ratio 56.6 (24.2-88.9). QUADAS 2 assessment showed intermediate/high risk of bias in a large proportion of the studies $(52-78 \%$ across the domains). No difference was present in sensitivity or specificity between subgroups when studies were split based on CT technique, sample size, nodule size, or publication date.

Conclusion: DCE-CT has a high diagnostic accuracy for the diagnosis of pulmonary nodules although study quality was indeterminate in a large number of cases.

\title{
Other:
}

The meta-analysis was prospectively registered on PROSPERO (CRD42018112215). The trial was funded by NIHR HTA grant 09-22-117.

\section{Key words}


Solitary Pulmonary Nodule; Meta-Analysis; Multidetector Computed Tomography; Contrast Media; Lung Cancer

Key points

- The pooled accuracy results were sensitivity $95.1 \%$ and specificity $73.8 \%$ although individual studies showed wide ranges of values.

- This is comparable to the results of previous meta-analyses of PET/CT (Positron emission tomography/computed tomography) diagnostic accuracy for the diagnosis of solitary pulmonary nodules.

- Robust direct comparative accuracy and cost effectiveness studies are warranted to determine the optimal use of DCE-CT and PET/CT in the diagnosis of SPNs.

\section{Abbreviations}

DCE-CT: Dynamic contrast enhanced computed tomography

PET: $\quad$ Positron emission tomography

SPN: $\quad$ Solitary pulmonary nodules

SROC: $\quad$ Summary receiver operator characteristic

PLR: $\quad$ Positive likelihood ratio

NLR: $\quad$ Negative likelihood ratio

DOR: $\quad$ Diagnostic odds ratio

HU: $\quad$ Hounsfield units

QUADAS: Quality Assessment of Diagnostic Accuracy Studies 


\section{INTRODUCTION}

Despite significant advances in the diagnosis and treatment of lung cancer, it remains the leading cause of cancer mortality [1]. Although only a proportion of patients with lung cancer present with a solitary pulmonary nodule (SPN) on diagnostic imaging tests, this is an important group as an SPN can represent early stage lung cancer, with higher survival rates following surgical resection than larger lesions [2]. However, not all SPNs turn out to be lung cancer and the accurate characterisation of SPNs is an ongoing diagnostic challenge with significant associated health costs [3]. With the adoption of low dose Computed Tomography (CT)-based lung cancer screening programmes in many countries, the number of patients with a SPN requiring further investigation is likely to increase substantially [4].

An SPN is defined as a single pulmonary lesion less than $30 \mathrm{~mm}$ in size [5]. Positron emission tomography with computed tomography (PET/CT) is currently the recommended test for the investigation of an indeterminate SPN $\geq 8 \mathrm{~mm}$, particularly when a biopsy is not possible [6, 7]. However PET/CT is only available in specialist centres, with more limited availability than $\mathrm{CT}$, which can make access more difficult for an older population with a high burden of co-morbidities $[8,9]$. In addition, PET/CT is both time consuming and expensive relative to other non-invasive imaging modalities such as $\mathrm{CT}$. Where PET/CT measures the metabolism within the tissue of interest, dynamic contrast enhanced CT (DCE-CT) allows measurement of the vascularity of the tissue [10]. The degree of enhancement on DCE-CT has been shown to correlate well with grade of lung cancer and the vessel density in the tumour $[11,12]$. DCE-CT can be performed on most modern CT machines in current use and is therefore potentially readily accessible to patients. Furthermore DCE-CT could potentially be performed at the same CT examination at which the pulmonary nodule is found. Early work suggested a high diagnostic accuracy for DCE-CT, however this previous analysis incorporated a relatively small number of studies [13].

The aim of this systematic review of the literature and meta-analysis was to determine the diagnostic performance of dynamic contrast enhanced computed tomography (DCE-CT) for the differentiation of malignant from benign pulmonary nodules. 


\section{MATERIALS AND METHODS}

The study was prospectively enrolled in PROSPERO (CRD42018112215). The study has been reported in accordance with the Preferred Reporting Items for a Systematic Review and Metaanalysis of Diagnostic Test Accuracy Studies (PRISMA-DTA) statement [14].

The population of interest were those with a solitary pulmonary nodule undergoing a dynamic contrast enhanced CT as part of a work-up to determine the malignant or benign status of the nodule. The inclusion criteria were studies examining solitary pulmonary nodules being worked up for malignancy, and excluded those which included participants $<18$ years old, and those with pure ground glass nodules. The intervention of interest was dynamic contrast enhanced computed tomography. Computed tomographic scans were included as long as there was a minimum of both a pre-contrast and post contrast enhanced $\mathrm{CT}$ dataset for the quantification of the degree of enhancement. The gold standard against which the test was examined was required to be histological diagnosis of malignancy obtained from either needle biopsy or surgical resection, with benign status confirmed either histologically or with follow-up imaging showing no growth at 2 years or resolution. We considered both prospective and retrospective diagnostic accuracy studies which contained sufficient data to construct contingency tables in order to assess true positive, false positive, true negative and false negative results.

To identify articles of interest for review, Ovid MEDLINE and EMBASE were searched for published studies from their inception until October 2018 on the diagnostic accuracy of DCE-CT in the characterization of pulmonary nodules. The full search strategy is documented in Supplementary Table S1. Titles and abstracts of studies retrieved using the search strategy and those from additional sources were all independently screened by two reviewers (J.W.M and S.J, both with 1 year experience) to identify studies that potentially met the inclusion criteria outlined above. The full text of these potentially eligible studies were retrieved and independently reviewed by the two reviewers to assess for eligibility. Where there was a disagreement between the reviewers, a consensus was reached through discussion. The references of the retrieved full text articles were screened for further articles of interest and if any articles were found these were retrieved if they had not been previously identified with the original search strategy.

A single reviewer (J.W-M) used a standardised, pre-piloted form to extract data from the included studies for assessment of study quality and evidence synthesis. Extracted information included study population and participant demographics and baseline characteristics; details of the CT scanning hardware, scanning technique, and diagnostic threshold used; study methodology; nodule size range and eventual diagnosis; diagnostic accuracy metrics; radiation dose. 
Two review authors (J.W-M and S.J) independently assessed the risk of bias in the included studies through the use of the second version of the Quality Assessment of Diagnostic Accuracy Studies (QUADAS-2) questionnaire [15]. Discordance in the scoring of bias between the two reviewers were resolved by a third review author (L-M.D).

Three deviations occurred from the original pre-registered protocol. A size threshold was not prespecified in the original protocol, yet upon the literature review it became apparent that the upper size limit included varied markedly between studies. Although the Fleischner and BTS guidelines state that the upper limit of an SPN is $30 \mathrm{~mm}$, we allowed up to $40 \mathrm{~mm}$ for the purpose of this analysis due to the high quality of many of the studies using this threshold, and the granularity it would provide the review. However, an analysis was performed to compare studies with and without nodules above $30 \mathrm{~mm}$ as described in the statistical section. While our original protocol called for the analysis of solitary pulmonary nodules, we found that although several studies recruited cases based on the detection of a solitary pulmonary nodule, if an additional nodule was detected at the time of the index test, they included, analysed and followed up both lesions. Despite not being strictly 'solitary' pulmonary nodule studies these were included in the analysis as they reflect routine clinical practice where a second smaller nodule is identified when CT is performed following detection of a nodule on chest radiograph. Some studies reported average follow-up of the nodules detected on $\mathrm{CT}$, rather than a minimal follow-up period. Cancellation of follow-up after resolution of the nodule in the case of infectious/inflammatory nodules would reduce the mean length of follow-up below the pre-stated 2 year minimum, yet nodules are considered benign if they resolve. Therefore these studies were included in the meta-analysis. They were however classed as being at high risk of bias with regards to their application of the reference standard on the QUADAS-2 questionnaire due to the uncertainty about the minimum length of follow-up in stable nodules. The impact of this on the results was analysed as described below.

\section{Statistical analysis}

Numbers of true positives, false positives, true negatives and false negatives were extracted from the studies and used to form $2 \times 2$ contingency tables which were used to derive sensitivity, specificity, positive likelihood ratio (PLR), negative likelihood ratio (NLR) and diagnostic odds ratio (DOR). Results were pooled using the Ime4 package within R (RStudio Version 1.1.463, RStudio, Inc.) to perform a bivariate binomial random effects meta-analysis [16]. This uses a binary (logit) generalized linear mixed model fit by maximum likelihood (using a laplace approximation). Bivariate summary receiver operator characteristic (SROC) curves were constructed using the bivariate random effects model outputs to populate the SROC plot within Review Manager Version 5.3. (The 
Cochrane Collaboration). To identify potential sources of heterogeneity, we stratified a secondary analysis into subgroups according to characteristics such as sample size, lesion size, risk of bias (low versus high/indeterminate), diagnostic thresholds, whether the diagnostic threshold was prospectively set and year of publication. These were included as covariates, in turn, in a metaregression analysis, with analysis of statistical significance between models performed using a likelihood ratio test of nested models. For sample size, the threshold at which to split the data was arbitrarily set at 100 to represent larger samples that were less likely to be prone to bias due to outliers. For mean nodule size the sample was split at $20 \mathrm{~mm}$ to provide a reasonable split of the data. For maximum nodule size the data was split based on whether the study included nodules $>30 \mathrm{~mm}$, as the $30 \mathrm{~mm}$ diameter is considered by most guidelines as the upper threshold for a lesion to be called a nodule, after which it is considered to be a mass. Effect of publication date was examined by splitting on the median (2008), with studies published in the last decade considered to be more representative of modern CT technology. In studies reporting the diagnostic accuracy of multiple thresholds, the optimal threshold was used in the primary analysis. In the secondary analyses examining different thresholds, studies were included in each sub group analysis where they had reported the threshold of interest. Thresholds with $\leq 2$ studies reporting the same threshold were not considered for this secondary analysis. To test for study publication bias and heterogeneity a Galbraith plot was created to examine the interaction between the efficient score and variance, with the Harbord test used to test for funnel plot asymmetry [17]. All statistical analysis was performed using RStudio. Forest plots and SROC curves were generated using RevMan.

\section{RESULTS}

Of 3028 potential papers identified by the literature review, 22 were included which met the inclusion and exclusion criteria. An additional study was located from the references of the included papers resulting in 23 studies in the final analysis. Figure 1 details the flow of the studies identified, screened for eligibility, and the reasons for study exclusion.

23 studies were included, incorporating the results from 2397 patients with 2514 nodules. Of these 1389/2514 (55.3\%) were malignant. The studies were predominantly retrospective single centre studies, performed in a wide range of countries and settings (Table 1). The dynamic contrast enhanced CT protocol varied widely from study to study, from the injection rate to the scan timing to the tube settings (Table 2). 18 studies were performed using mono-energetic (routine) CT with 
regular interval imaging, and 5 were performed using CT perfusion techniques. The injection techniques included a standardised volume bolus and injection rate; adjusting the contrast volume to the weight of the patient; or adjusting the injection rate to the weight of the patient. Image acquisition ranged from 3 volume acquisitions at different phases of the contrast injection to 32 separate acquisitions. Most studies utilised an enhancement subtraction technique, taking the phase with the maximum nodule attenuation and subtracting the baseline attenuation to calculate the degree of enhancement. However several studies utilised the slope of the enhancement curve or the area under the enhancement curve.

The results of the QUADAS-2 bias and applicability assessment are summarised in Figure 2 whilst Table 3 documents the individual bias scores for the seven domains for all included studies. Bias in patient selection was unclear in a large number $(14 / 23,61 \%)$ of studies due to a lack of reporting of the sampling of patients for the diagnostic test accuracy evaluation, with many retrospective studies not clearly documenting whether consecutive cases were included or not. Risk of bias in the index test was high in a large number of studies $(12 / 23,52 \%)$ due to a lack of pre-specification of the intended threshold to be used, and in several studies multiple techniques of enhancement of quantification were used simultaneously (including but not limited to absolute contrast enhancement, relative contrast enhancement, wash-in, wash-out, wash in and wash-out, and area under the enhancement curve). Bias regarding the reference standard was unclear in the majority of studies $(18 / 23,78 \%)$, with the blinding of the reference standard to the index test infrequently reported. Flow and timing had a similar high rate frequency of uncertainty bias $(15 / 23,65 \%)$, with the delay between the index test and reference standard infrequently reported. Concerns regarding the applicability of the included studies to the review question were low for the majority of the studies (Figure 2).

The results of the individual studies sensitivities and specificities are collated in a forest plot in Figure 3 , with all studies reporting a per nodule diagnostic accuracy. The pooled analysis of the 24 studies is reported in Table 4. The pooled sensitivity and specificity were $94.8(95 \% \mathrm{Cl} 91.5 ; 96.9)$ and 75.5 $(95 \% \mathrm{Cl} 69.4$; 80.6) respectively (see SROC plot in Figure 4), with a positive and negative likelihood ratio of $3.86(2.99 ; 4.74)$ and $0.07(0.03 ; 0.10)$, and a diagnostic odds ratio of $56.6(24.2 ; 88.9)$. Only two distinct enhancement thresholds were reported by $>2$ studies with the pooled analysis for each of these reported in Table 4. Of these, a threshold of $<20$ Hounsfield units (HU) enhancement for the differentiation of a malignant from a benign nodule had the highest diagnostic odds ratio of 
142.5 (95\% Cl -36.4; 321.3), maintaining a high sensitivity of $98.3 \%(95 \% \mathrm{Cl} 95.1 ; 99.4)$ and moderate specificity of $71.0 \%(95 \% \mathrm{Cl} 63.1 ; 77.8)$ (Table 4).

The Galbraith plot (Figure 5) demonstrated multiple studies falling outwith the $95 \%$ confidence intervals consistent with a significant inter-study heterogeneity in findings, but there was not any significant asymmetry in the plot $(p=0.90)$ to suggest publication bias. A formal analysis of the degree of heterogeneity was not performed as per the Cochrane Collaborations recommendations on diagnostic test accuracy meta-analysis; however factors that may have contributed to the heterogeneity were examined (Table 5). Studies with a low risk for reference standard bias demonstrated a higher sensitivity and with equivalent specificity compared with studies with intermediate/high risk $(p=0.044)$. However only two studies - both conducted by the same group were considered to be at low risk. Studies conducted pre-2008 had slightly higher sensitivity and specificity compared with those from 2008 onwards although this did not reach statistical significance $(p=0.07)$. The CT technique (mono-energetic, versus perfusion) did not affect diagnostic accuracy $(p=0.42)$. No difference was present between subgroups when studies were split based on sample size, mean or maximum nodule size, threshold prospectively or retrospectively set, or the presence of patient selection bias, index test bias or flow and timing bias ( $p>0.1$ for all). In particular, there was no significant difference in the pooled sensitivity or specificity between studies that only included nodules $\leq 30 \mathrm{~mm}$ (and therefore meet current definitions of SPNs) compared with those that included larger nodules up to $40 \mathrm{~mm}$ in size $(p=0.07$ for between group differences in sensitivity and specificity). 


\section{DISCUSSION}

This meta-analysis demonstrates a high sensitivity and moderate specificity for dynamic contrast enhanced computed tomography for the diagnosis of solitary pulmonary nodules with a pooled sensitivity and specificity of $94.8 \%$ and $75.5 \%$ respectively. However the study quality was indeterminate in a significant proportion of the studies with only one multi-centre study and a large number of small single centre studies. While the analysis shows promising results for the technique, the low quality of the included studies must be taken into account and further carefully designed high quality multi-centre studies are required.

The current Fleischner guidelines for further investigation and management of indeterminate solitary pulmonary nodules call for either PET/CT or biopsy if the nodule is $>8 \mathrm{~mm}$ [6], with dynamic contrast enhanced computed tomography not mentioned in the diagnostic pathway despite inclusion of the technique in the 2005 version of the guidelines [5]. The British Thoracic Society guidelines state that dynamic contrast enhanced computed tomography should not be used where positron emission tomography is available although it is acknowledged that there is little evidence to support this beyond the historical prerogative of PET/CT [7]. A recent meta-analysis of PET/CT including 20 studies with 1,557 participants reported a sensitivity and specificity of $89 \%$ and $70 \%$, and a diagnostic odds ratio (DOR) of 22 [39]. These results are similar to the DCE-CT results obtained in this meta-analysis with the 23 studies including 2397 participants, demonstrating a pooled sensitivity, specificity and DOR of $95 \%, 76 \%$ and 57 respectively. This suggests that DCE-CT could replace PET/CT as an equivalent diagnostic technique. Currently there are a limited number of studies directly comparing DCE-CT with PET/CT, precluding the ability to perform a meta-analytic comparison. Ohno et al. compared DCE-CT with both PET/CT and dynamic contrast enhanced MRI in a single centre study of 198 patients, and found that DCE-CT out performed both MRI and PET/CT in specificity and accuracy [10]. This contradicted results of $Y i$ et al. who found, in a single centre study of 119 participants, that PET/CT was more sensitive with equal specificity to that of DCE-CT [40]. Thus further work is required to directly compare these two modalities. Another technique that has a growing body of evidence is that of diffusion weighted MRI (DW-MRI). While PET/CT examines metabolism and DCE-CT measures perfusion, DW-MRI quantifies the movement of water within the lesion. A recent meta-analysis of diffusion-weighted MRI for the diagnosis of indeterminate solitary pulmonary nodules has suggested superiority of this technique compared to PET/CT with a pooled sensitivity, specificity and DOR of $83 \%, 91 \%$ and 50 respectively for diffusion weighted MRI compared with $78 \%, 81 \%$ and 15 for PET/CT [41]. Furthermore, dynamic contrast enhancement can also be quantified on MRI in the same examination as the assessment of diffusion [42]. Given the differing nature of the 3 parameters in question, further research is needed to determine whether 
the information from perfusion, diffusion and metabolism are complimentary or duplicative in improving diagnostic accuracy.

The equivalent sensitivity, specificity and accuracy in this meta-analysis of DCE-CT compared to previous meta-analysis of PET/CT provides supportive evidence for consideration of incorporation of DCE-CT into the diagnostic pathway of pulmonary nodules. CT machines are more commonly found and more readily accessible in hospital settings than PET/CT. A dynamic contrast examination is very similar to a standard contrast CT procedure which is commonly undertaken at all hospitals and requires no additional equipment. A PET/CT examination requires the injection of a radioactive substrate, which needs to be delivered reliably to centres undertaking PET examinations. The requirement of such a supply chain can have significant impact on service flexibility and can result in scan cancellations when there is disruption or delay in delivery of the radioactive agent [43]. Future studies examining whether certain subgroups of pulmonary nodules (such as small size) or those found in patients with different risk profiles and likelihood of malignancy may have more to gain from a DCE-CT examination than PET/CT are also required. Similarly a tiered approach using DCE-CT as the first diagnostic test and gatekeeper to PET/CT may allow for a more nuanced work-up approach utilising the strengths of both techniques. Such an approach has been shown to be a cost effective approach to the diagnosis of SPNs [44]. Robust direct comparative accuracy of DCE-CT and $\mathrm{PET} / \mathrm{CT}$ in the same population and cost effectiveness studies are warranted to test the various diagnostic pathways.

There are several limitations with the current meta-analysis. The quality of the included studies was frequently indeterminate due to lack of reporting of key metrics. The studies were almost exclusively single centre and frequently retrospective, both of which are likely to amplify the apparent diagnostic accuracy of the technique. In addition the dynamic contrast acquisition technique and the metrics for the quantification of the enhancement were heterogeneous throughout the studies. Whilst these factors did not appear to have an impact on the accuracy of meta-regression, a standardised acquisition and analysis technique should be agreed upon to improve reproducibility and facilitate comparison between trials thereby allowing more widespread adoption. The observed rate of malignancy in the included studies is relatively high (55\%). Whilst this is consistent with previous meta-analysis of MRI and PET in SPNs $[39,41]$, it is substantially higher when compared to screening detected SPNs such as in the National Lung Screening Trial $(15.0 \%$ malignancy in $10-30 \mathrm{~mm}$ nodules) and NELSON trial (15.2\% malignancy in nodules $>10 \mathrm{~mm})[45,46]$. Previous work has shown the sensitivity of a technique to be relatively robust to disease prevalence and for the specificity to increase with falling prevalence [47]. It can be postulated that the diagnostic accuracy of DCE-CT would be similar, or even further improved, in a screening population. 
In conclusion we have found a high diagnostic accuracy of DCE-CT for the diagnosis of pulmonary nodules although study quality was poor or indeterminate in a large number of cases. The diagnostic accuracy is comparable to a recent meta-analysis of PET/CT suggesting that DCE-CT may compliment or augment the current diagnostic pathway used for the investigation of solitary pulmonary nodules.

\section{ACKNOWLEDGEMENTS}

The study was funded by NIHR Health Technology Assessment programme, project number 0922 117 and supported by the NIHR Cambridge Biomedical Research Centre. AC is part-funded by the National Institute for Health Research Collaboration for Leadership in Applied Health Research and Care North West Coast (NIHR CLAHRC NWC). FJG is part-funded by an NIHR Senior Investigator award. RCR is part funded by Cambridge Biomedical Research Centre and Cancer Research UK Cambridge Centre. NRQ is part funded by Cambridge Biomedical Research Centre. The funders had no role in the design, analysis or write-up of the study. The views expressed are those of the authors and not necessarily those of the NHS, NIHR or Department of Health. 
TABLES

Table 1: Summary of the study design and baseline characteristics of those included in the metaanalysis

$\mathrm{B}=$ Benign; $\mathrm{M}=$ Malignant; $\mathrm{FU}=$ Follow-up

* Value not provided for whole cohort and could not be calculated based on sub-group reported values.

Table 2: Summary of the CT acquisition protocols and measurements of the studies included in the meta-analysis

$\wedge$ Scan type as follows: 1 - mono-energetic dynamic contrast enhanced computed tomography; 2 - computed tomography perfusion.

\& Hounsfield unit difference between baseline and peak enhancement unless otherwise stated

Table 3: Table of the QUADAS-2 components for each of the individual studies

(i)Low Risk $\quad$ OHigh Risk ? Unclear Risk

Table 4: Diagnostic performance of Dynamic contrast enhanced CT for the evaluation of pulmonary nodules.

$\mathrm{HU}=$ Hounsfield units; NLR = Negative likelihood ratio; PLR = Positive likelihood ratio; DOR = Diagnostic odds ratio 
Table 5: Subgroup Analyses of the Diagnostic Performance of DCE-CT for Evaluation of Indeterminate Pulmonary Lesions

* Mean lesion size not reported in 3 studies, and reported as volumes rather than diameter in 3 studies, max lesion size reported as volumes in 3 studies.

$\mathrm{CTP}=$ Computer tomography perfusion; DECT = Dual energy dynamic contrast enhanced computer tomography; $\mathrm{MECT}=$ Mono-energetic dynamic contrast enhanced computer tomography. 
FIGURES

Figure 1: Flow diagram of the articles identified by the literature search, screened for eligibility, and included in the final study.

$\mathrm{CT}$ = Computed tomography; $\mathrm{MRI}=$ Magnetic resonance imaging; $\mathrm{PET} / \mathrm{CT}=$ Positron Emission Tomography; SPECT = Single positron emission computed tomography.

Figure 2: QUADAS scoring summary of the included studies

Figure 3: Forest plot of the included studies

Studies listed by first author and year of publication

$\mathrm{Cl}=$ Confidence intervals $; \mathrm{FN}=$ False negative; $\mathrm{FP}=$ False positive $; \mathrm{TN}=$ True negative $; \mathrm{TP}=$ True positive

Figure 4: Bivariate SROC curve of the included studies. The white circles indicate each individual study whilst the black circle indicates the summary point. The dotted line is the $95 \%$ confidence region for the summary operating point, whilst the dashed line is the $95 \%$ prediction region (which is the confidence region for a forecast of the true sensitivity and specificity in any future study).

Figure 5: Galbraith plot examining inter-study heterogeneity for publication bias by incorporating the effect size of each study compared with the pooled analysis. The y-axis represents the test statistics (effect/standard error of the estimate) of each study, which are expected to fall within 2 units of the pooled effects for $95 \%$ of the studies. The $x$-axis plots $1 /$ standard error of the pooled study estimate. 


\section{REFERENCES}

1. Siegel RL, Miller KD, Jemal A (2015) Cancer statistics, 2015. CA Cancer J Clin 65:5-29. https://doi.org/10.3322/caac.21254

2. Tanner NT, Dai L, Bade BC, et al (2017) Assessing the generalizability of the national lung screening trial: Comparison of patients with stage 1 disease. Am J Respir Crit Care Med 196:602-608. https://doi.org/10.1164/rccm.201705-09140C

3. Barnett PG, Ananth L, Gould MK (2010) Cost and Outcomes of Patients With Solitary Pulmonary Nodules Managed With PET Scans. Chest 137:53-59. https://doi.org/10.1378/chest.08-0529

4. National Lung Screening Trial Research Team, Aberle DR, Adams AM, et al (2011) Reduced lung-cancer mortality with low-dose computed tomographic screening. N Engl J Med 365:395-409. https://doi.org/10.1056/NEJMoa1102873

5. MacMahon H, Austin JHM, Gamsu G, et al (2005) Guidelines for Management of Small Pulmonary Nodules Detected on CT Scans: A Statement from the Fleischner Society. Radiology 237:395-400. https://doi.org/10.1148/radiol.2372041887

6. MacMahon H, Naidich DP, Goo JM, et al (2017) Guidelines for Management of Incidental Pulmonary Nodules Detected on CT Images: From the Fleischner Society 2017. Radiology 284:228-243. https://doi.org/10.1148/radiol.2017161659

7. Callister MEJ, Baldwin DR, Akram AR, et al (2015) British Thoracic Society guidelines for the investigation and management of pulmonary nodules. Thorax 70 Suppl 2:ii1-ii54. https://doi.org/10.1136/thoraxjnl-2015-207168

8. Matsumoto M, Koike S, Kashima S, Awai K (2015) Geographic distribution of CT, MRI and PET devices in Japan: A longitudinal analysis based on national census data. PLoS One 10:1-12. https://doi.org/10.1371/journal.pone.0126036

9. Parker L, Levin DC, Frangos A, Rao VM (2010) Geographic variation in the utilization of noninvasive diagnostic imaging: National medicare data, 1998-2007. Am J Roentgenol 194:1034-1039. https://doi.org/10.2214/AJR.09.3528

10. Ohno $\mathrm{Y}$, Nishio $\mathrm{M}$, Koyama $\mathrm{H}$, et al (2015) Solitary pulmonary nodules: Comparison of dynamic first-pass contrast-enhanced perfusion area-detector $\mathrm{CT}$, dynamic first-pass contrast-enhanced MR imaging, and FDG PET/CT. Radiology 274:563-75. https://doi.org/10.1148/radiol.14132289 
11. Yi CA, Lee KS, Kim EA, et al (2004) Solitary Pulmonary Nodules: Dynamic Enhanced MultiDetector Row CT Study and Comparison with Vascular Endothelial Growth Factor and Microvessel Density. Radiology 233:191-199. https://doi.org/10.1148/radiol.2331031535

12. BAI R, CHENG X, QU H, et al (2009) Solitary pulmonary nodules: comparison of multi-slice computed tomography perfusion study with vascular endothelial growth factor and microvessel density. Chin Med J (Engl) 122:541-547. https://doi.org/10.3760/cma.j.issn.0366-6999.2009.05.011

13. Cronin P, Dwamena BA, Kelly AM, Carlos RC (2008) Solitary Pulmonary Nodules: Metaanalytic Comparison of Cross-sectional Imaging Modalities for Diagnosis of Malignancy. Radiology 246:772-782. https://doi.org/10.1148/radiol.2463062148

14. Mclnnes MDF, Moher D, Thombs BD, et al (2018) Preferred Reporting Items for a Systematic Review and Meta-analysis of Diagnostic Test Accuracy Studies. Jama 319:388. https://doi.org/10.1001/jama.2017.19163

15. Whiting PF, Rutjes AWS, Westwood ME, et al (2011) Research and Reporting Methods Accuracy Studies. Ann Intern Med 155:529-536. https://doi.org/10.7326/0003-4819-155-8201110180-00009

16. Bates D, Mächler M, Bolker B, Walker S (2015) Fitting Linear Mixed-Effects Models Using Ime4. J Stat Softw 67:. https://doi.org/10.18637/jss.v067.i01

17. Harbord RM, Egger M, Sterne JAC (2006) A modified test for small-study effects in metaanalyses of controlled trials with binary endpoints. Stat Med 25:3443-3457. https://doi.org/10.1002/sim.2380

18. Swensen J, Cortese A, Morin L, et al (1992) Solitary Pulminary Nodule: CT Evaluation of Enhancement with lodinated Contrast Material - A Preliminary Report. Radiology 182:343347

19. Swensen J, Brown R, Colby V, Weaver L (1995) Thoracic Nodules : CT Evaluation with lodinated Contrast. Radiology

20. Yamashita K, Matsunobe S, Tsuda T, et al (1995) Solitary pulmonary nodule: preliminary study of evaluation with incremental dynamic CT. Radiology 194:399-405. https://doi.org/10.1148/radiology.194.2.7824717

21. Swensen SJ, Brown LR, Colby T V, et al (1996) Lung nodule enhancement at CT: prospective findings. Radiology 201:447-55. https://doi.org/10.1148/radiology.201.2.8888239 
22. Potente G, lacari V, Caimi M (1997) The challenge of solitary pulmonary nodules: HRCT evaluation. Comput Med Imaging Graph 21:39-46

23. Zhang M, Kono M (1997) Solitary pulmonary nodules: evaluation of blood flow patterns with dynamic CT. Radiology 205:471-8. https://doi.org/10.1148/radiology.205.2.9356631

24. Swensen SJ, Viggiano RW, Midthun DE, et al (2000) Lung Nodule Enhancement at CT: Multicenter Study. Radiology 214:73-80. https://doi.org/10.1148/radiology.214.1.r00ja1473

25. Kim JH, Kim HJ, Lee KH, et al (2004) Solitary pulmonary nodules: a comparative study evaluated with contrast-enhanced dynamic MR imaging and CT. J Comput Assist Tomogr 28:766-775. https://doi.org/00004728-200411000-00007 [pii]

26. Orlacchio A, Schillaci O, Antonelli L, et al (2007) Nodulo polmonare solitario: Caratterizzazione morfologico-metabolica mediante imaging integrato TCms/FDG-PET. Radiol Medica 112:157173. https://doi.org/10.1007/s11547-007-0132-x

27. Lee KS, Yi CA, Jeong SY, et al (2007) Solid or partly solid solitary pulmonary nodules: Their characterization using contrast wash-in and morphologic features at helical CT. Chest 131:1516-1525. https://doi.org/10.1378/chest.06-2526

28. Ohno Y, Koyama H, Takenaka D, et al (2008) Dynamic MRI, dynamic multidetector-row computed tomography (MDCT), and coregistered 2-[fluorine-18]-fluoro-2-deoxy-D-glucosepositron emission tomography (FDG-PET)/CT: Comparative study of capability for management of pulmonary nodules. J Magn Reson Imaging 27:1284-1295. https://doi.org/10.1002/jmri.21348

29. Eun JC, Gong YJ, Young MH, et al (2008) Solitary pulmonary nodule on helical dynamic CT scans: Analysis of the enhancement patterns using a Computer-Aided Diagnosis (CAD) system. Korean J Radiol 9:401-408. https://doi.org/10.3348/kjr.2008.9.5.401

30. Bayraktaroglu S, Savaş R, Basoglu ÖK, et al (2008) Dynamic computed tomography in solitary pulmonary nodules. J Comput Assist Tomogr 32:222-227. https://doi.org/10.1097/RCT.0b013e318136e29d

31. Jiang NC, Han P, Zhou CK, et al (2009) Dynamic enhancement patterns of solitary pulmonary nodules at multi-detector row $\mathrm{CT}$ and correlation with vascular endothelial growth factor and microvessel density. Chin J Cancer 28:164-169

32. Dabrowska M, Zukowska M, Krenke R, et al (2010) Simplified method of dynamic contrastenhanced computed tomography in the evaluation of indeterminate pulmonary nodules. 
Respiration 79:91-6. https://doi.org/10.1159/000213760

33. Li Y, Yang ZG, Chen TW, et al (2010) First-pass perfusion imaging of solitary pulmonary nodules with 64-detector row CT: Comparison of perfusion parameters of malignant and benign lesions. Br J Radiol 83:785-790. https://doi.org/10.1259/bjr/58020866

34. Ohno Y, Koyama H, Matsumoto K, et al (2011) Differentiation of malignant and benign pulmonary nodules with quantitative first-pass 320-detector row perfusion CT versus FDG PET/CT. Radiology 258:599-609. https://doi.org/10.1148/radiol.10100245

35. Ohno $\mathrm{Y}$, Nishio $\mathrm{M}$, Koyama $\mathrm{H}$, et al (2013) Comparison of quantitatively analyzed dynamic area-detector CT using various mathematic methods with FDG PET/CT in management of solitary pulmonary nodules. Am J Roentgenol 200:593-602.

https://doi.org/10.2214/AJR.12.9197

36. Shu SJ, Liu BL, Jiang HJ (2013) Optimization of the scanning technique and diagnosis of pulmonary nodules with first-pass 64-detector-row perfusion VCT. Clin Imaging 37:256-264. https://doi.org/10.1016/j.clinimag.2012.05.004

37. Ribeiro SM, Ruiz RL, Yoo HHB, et al (2013) Proposal to utilize simplified swensen protocol in diagnosis of isolated pulmonary nodule. Acta radiol 54:757-764.

https://doi.org/10.1177/0284185113481695

38. Ye X-D, Ye J-D, Yuan Z, et al (2014) Dynamic CT of solitary pulmonary nodules: comparison of contrast medium distribution characteristic of malignant and benign lesions. Clin TransI Oncol 16:49-56. https://doi.org/10.1007/s12094-013-1039-8

39. Li ZZ, Huang YL, Song HJ, et al (2018) The value of 18 F-FDG-PET/CT in the diagnosis of solitary pulmonary nodules: A meta-analysis. Med (United States) 97:.

https://doi.org/10.1097/MD.0000000000010130

40. Yi C a, Lee KS, Kim B-T, et al (2006) Tissue characterization of solitary pulmonary nodule: comparative study between helical dynamic CT and integrated PET/CT. J Nucl Med 47:443450

41. Basso Dias A, Zanon M, Altmayer S, et al (2019) Fluorine 18-FDG PET/CT and Diffusionweighted MRI for Malignant versus Benign Pulmonary Lesions: A Meta-Analysis. Radiology 290:525-534. https://doi.org/10.1148/radiol.2018181159

42. Zhou SC, Wang YJ, Ai T, et al (2019) Diagnosis of solitary pulmonary lesions with intravoxel incoherent motion diffusion-weighted MRI and semi-quantitative dynamic contrast-enhanced 
MRI. Clin Radiol 74:409.e7-409.e16. https://doi.org/10.1016/j.crad.2018.12.022

43. Ducharme J, Goertzen AL, Patterson J, Demeter S (2009) Practical aspects of 18 F-FDG PET when receiving 18 F-FDG from a distant supplier. J Nucl Med Technol 37:164-170. https://doi.org/10.2967/jnmt.109.062950

44. Comber LA, Keith CJ, Griffiths M, Miles KA (2003) Solitary pulmonary nodules: Impact of quantitative contrast-enhanced CT on the cost-effectiveness of FDG-PET. Clin Radiol 58:706711. https://doi.org/10.1016/S0009-9260(03)00166-1

45. Church TR, Black WC, Aberle DR, et al (2013) Results of initial low-dose computed tomographic screening for lung cancer. N Engl J Med 368:1980-1991. https://doi.org/10.1056/NEJMoa1209120

46. Horeweg N, van Rosmalen J, Heuvelmans MA, et al (2014) Lung cancer probability in patients with CT-detected pulmonary nodules: A prespecified analysis of data from the NELSON trial of low-dose CT screening. Lancet Oncol 15:1332-1341. https://doi.org/10.1016/S14702045(14)70389-4

47. Leeflang MMG, Rutjes AWS, Reitsma JB, et al (2013) Variation of a test's sensitivity and specificity with disease prevalence. Cmaj 185:537-544. https://doi.org/10.1503/cmaj.121286 